\title{
Isolamento social em tempos de pandemia por covid- 19: impactos na saúde mental da população
}

\section{Social isolation in pandemic times by covid-19: impacts on the men- tal health of the population}

\author{
Ítalo Arão Pereira Ribeiro' • Mariza Ozório da Rocha² • Denilson César Lopes Cunha ${ }^{3}$ \\ Adriana da Silva Araújo ${ }^{4} \bullet$ Isadora Nunes Amaral ${ }^{5} \bullet$ Letícia Lacerda Marques $^{6} \bullet$ Morgana Boaventura Cunha $^{7}$
}

Desde dezembro de 2019 o mundo vem enfrentando as duras consequências da pandemia de SARS-COV-2/COVID-19, classificada como emergência de saúde pública de interesse internacional pela Organização Mundial da Saúde (OMS) (1). Nesse sentido, devido à magnitude do problema, medidas como o isolamento social e a quarentena foram adotadas como principais recomendações de defesa e combate a esta forte ameaça à saúde mundial.

Entretanto, períodos prolongados de isolamento social, somados ao medo de serem infectados por um vírus potencialmente infeccioso cujos aspectos de origem, manejo e tratamento, ainda são poucos compreendidos, acabam afetando o bem-estar psicológico de muitas pessoas, desencadeando uma variedade de sintomas psicopatológicos a curto prazo, como: estresse, humor deprimido, irritabilidade, ansiedade, medo, raiva, insônia e outros; e a longo prazo, como aumento do risco de abuso de álcool, sintomas de transtorno de estresse pós-traumático e depressão ${ }^{(2-3)}$.

Pesquisas sobre os impactos do isolamento social na saúde mental causados pelo COVID-19 ainda são incipientes, porém, tais efeitos já foram observados em situações semelhantes, em quarentenas de pequenos grupos envolvendo epidemias com SARS-COV-I, HINI, Ebola e o surto da Síndrome Respiratória do Oriente Médio (MERS) ${ }^{(2)}$, ocorrida em 2015 na Coréia do Sul, no qual identificou que o nível de estresse diário e os sintomas do estresse pós-traumático podem ser fatores de risco para o desenvolvimento de depressão em pessoas submetidas à quarentena.

Da mesma forma, outros fatores psicológicos como medo, tensão, raiva e desconfiança podem surgir durante esse período ${ }^{(5)}$, estando estes danos potencialmente ligados aos casos de suicídios que se manifestão em períodos de isolamento(3). Sem mencionar, os refelexos dos impactos na econômia gerados pelo isolamento social prolongado, como dificuldades econômicas, aumento do desemprego, frustração, falta de suprimentos básicos, informações insuficientes e estigma, que também corroboram com a vulnerabilidade psicológica e deterioração da saúde mental(2).

Em tempos de COVID-19, várias atividades que antes faziam parte da rotina da população, principalmente as relacionadas às relações humanas, foram interrompidas, forçando a necessidade de novas adaptações, para as quais muitas pessoas não estavam preparadas ${ }^{(6)}$. Portanto, as repercussões derivadas do isolamento social sobre o bem-estar psicológico das pessoas devem ser discutidas, a fim de estimularem mais pesquisas sobre o assunto, bem como incentivar o desenvolvimento e a implementação de políticas públicas com ações voltadas não apenas à assistência dos efeitos físicos da doença, mas que atuem na prevenção e promoção da saúde mental em momentos como este.

I Enfermeiro, Doutorando em Enfermagem pelo Programa de Pós-Graduação em Enfermagem da Universidade Federal do Piauí (PPGEnf/UFPI).Teresina, Piauí, Brasil.E-mail: italoarao@ufpi.edu.br;

2 Enfermeira, Hospital Universitário João de Barros Barreto da Universidade Federal do Pará (HUJBB/UFPA). Belém, Pará, Brasil. E-mail: marizabn@hotmail.com;

3 Enfermeiro, Hospital Universitário João de Barros Barreto da Universidade Federal do Pará (HUJBB/UFPA). Belém, Pará, Brasil. E-mail:denilson.cesar@hotmail.com;

4 Enfermeira, Especialista em Saúde Pública pela Faculdade Integrada de Gestão e Meio Ambiente (FACIGMA). Maceió,Alagoas, Brasil. E-mail: adriana-enf20I@ hotmail.com;

5 Enfermeira, Especialista em Enfermagem do Trabalho e Saúde Ocupacional pela Universidade Cândido Mendes (UCAM). Maceió, Alagoas, Brasil.E-mail: Isadora.n.amaral@hotmail.com

6 Enfermeira, Hospital Universitário Professor Alberto Antunes da Universidade Federal de Alagoas (HUPAA/UFAL). Maceió, Alagoas, Brasil. E-mail: leticialacerda_05@hotmail.com

7 Enfermeira, Maternidade Escola Assis Chateaubriand da Universidade Federal do Ceará (MEAC/UFC). Ceará, Fortaleza, Brasil. E-mail: morgana.boaventura@ hotmail.com 


\section{REFERÊNCIAS}

I.Vasconcelos CSdaS, Feitosa IdeO, Medrado PLR, Brito APBde. O novo coronavirus e os impactos psicológicos da quarentena. Revista Desafios. 2020; 7(supl.): 75-80. https://doi. org/ I 0.20873/uftsuple2020-88|6

2.Afonso P. O Impacto da Pandemia COVID-I 9 na Saúde Mental. Revista Científica da Ordem dos Médicos. 2020; 33(I3): I. http://dx.doi.org/I 0.20344/amp. I 3877.

3. Schmidt B, Crepaldi MA, Bolze SDA, Neiva-Silva L, Demenech LM. Impactos na Saúde Mental e Intervenções Psicológicas
Diante da Pandemia do Novo Coronavírus (COVID-19). SciELO Preprints. 2020;00(00): I-26. 10.1590/SciELOPreprints.58 4. Kim H, Yoo S, Lee B, Lee S, Shin H. Psychiatric Findings in Suspected and Confirmed Middle East Respiratory Syndrome PatientsQuarantined in Hospital: A Retrospective Chart Analysis. Psychiatry Investigation. 2018 ; 15 (4):355360. 10.30773/pi.2017.10.25.I

5. Santos CF. Reflections about the impact of the SARS-COV-2/ COVID-19 pandemic on mental health. Braz J Psychiatry. 2020; 00(0): I. I0. I590/I5 I6-4446-2020-098।. 